\title{
A Video Capsule Attached to a Probe Can Be Used for Prolonged Stationary Endoscopic Monitoring
}

Endoscopic monitoring of the upper gastrointestinal tract could have an important role in the study of bleeding lesions or drug-induced changes in motility. In an attempt to achieve these aims, we assessed the feasibility of using a video capsule (Given Imaging, Yoqneam, Israel), bound to a flexible probe, to make prolonged stationary recordings of areas of interest $[1,2]$.

Following a Mason operation/bariatric surgery, a 47-year-old woman experienced vomiting due to tight pylorospasm. Balloon dilations led to transient symptomatic improvement; however the pylorospasm recurred. After the patient had given informed consent, we injected $25 \mathrm{U}$ of botulinum toxin into each quadrant of the prepyloric antrum [3]. Then, with the intention of monitoring the relaxation of the pylorus, we carried out the following procedure, with the patient under general anesthesia. A video capsule was fixed to the tip of an enteral feeding tube (Figure 1). A small wire loop and a long wire were fixed at the proximal and distal ends, respectively, of what was now termed the "capsule-probe." Using a biopsy forceps, the long wire was externalized at the proximal end of the operative channel of a video gastroscope (Olympus XP $160,5.9 \mathrm{~mm}$ ) and attached to its handle. The capsule-probe was introduced orally, parallel to the video gastroscope, and then unbound from it. The video gastroscope was reintroduced through a nostril, so that the small proximal wire loop could be grasped with the biopsy forceps, allowing the capsule-probe to be externalized via this nostril. The capsule-probe was fixed in place when the video capsule was facing the area of interest, as checked with the video gastroscope. Finally, the patient was connected to the recording system. The recording provided by the capsule-probe allowed observation of transient pyloric openings.

We have demonstrated the feasibility of placement and use of the capsule-probe, which allows prolonged recorded obser-

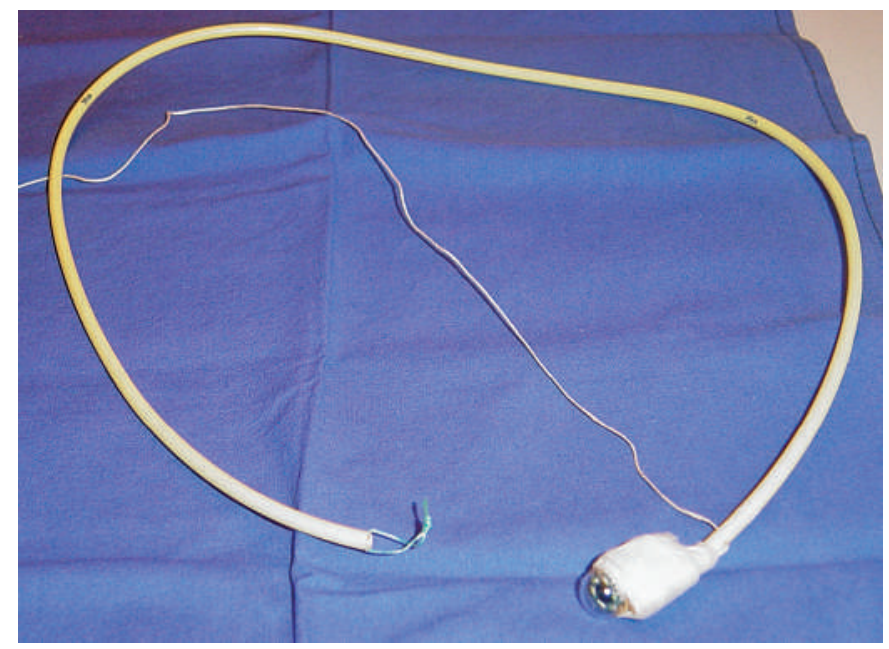

Figure 1 Video capsule bound to the probe (the "capsuleprobe“).

vation of specific parts of the upper digestive tract. It could be the first step towards endoscopic monitoring, a previously unreported concept. Such a technique could emerge with the development of a miniaturized video capsule, having the potential for transnasal use, with prolonged recording and immediate reading when coupled with a probe.

In our patient, the capsule-probe was used to study pyloric motility after injections of botulinum toxin. The recording did not allow a definitive conclusion about the efficacy of the treatment, although it did suggest that, prolonged endoscopic observation of motility is possible using a capsule-probe. However the most important potential applications of endoscopic monitoring might relate to upper gastrointestinal bleeding: first, the exact location of small intermittently bleeding lesions (for example Dieulafoy lesions) when their site is only approximately known; and second, the early detection of recurrent hemorrhage of identified bleeding lesions. The intensive care unit of the future might be equipped with screens showing real-time images transmitted from capsule-probes.

Competing interests: None

\section{Antonietti, G. Savoye, S. Lecleire, P. Hecketsweiler, E. Ben-Soussan} Endoscopic Gastroenterology Unit, Rouen University Hospital Charles Nicolle, Rouen, France

\section{References}

${ }^{1}$ Iddan G, Meron G, Glukhovsky A, Swain P. Wireless capsule endoscopy. Nature 2000; 405: 417

${ }^{2}$ Lewis BS, Swain P. Capsule endoscopy in the evaluation of patients with suspected small intestinal bleeding: results of a pilot study. Gastrointest Endosc 2002; 56: 349-353

${ }^{3}$ Wiesel PH, Schneider R, Dorta Get al. Botulinum toxin for refractory postoperative pyloric spasm. Endoscopy 1997; 29: 132

\section{Corresponding Author}

\section{Antonietti, M.D.}

Department of Gastroenterology

Rouen University Hospital Charles Nicolle 1 rue de Germont

76031 Rouen Cedex

France

Fax: $\quad+33-2-35151623$

E-mail: michel.antonietti@chu-rouen.fr 